Vida Jesenšek*

Universität Maribor
UDK [81'373.7:81'374]:81'33

DOI: 10.4312/linguistica.59.1.141-153

\title{
PHRASEOLOGIE UND PRAGMATIK
}

Sprache als Tätigkeit des Geistes und als sein Ausdruck in der Rede ist Sprache in der Kommunikation.

(Siegfried Heusinger)

\section{EINLEITUNG}

Die kommunikativ-pragmatische Wende in der Sprachwissenschaft stellte seit etwa 1970 eine funktionale Orientierung in der linguistischen Forschung in den Vordergrund; im Unterschied zu sprachhistorischen und/oder systemlinguistischen Herangehensweisen rückt in den Mittelpunkt der Betrachtung die Sprachverwendung samt ihrer Wirkung in unterschiedlichen kommunikativ-situativen Kontexten. Gleichermaßen gilt das Gesagte für die Erforschung der phraseologischen Ausdrucksweise: untersuchte man zu Anfängen der Phraseologie als selbstständige linguistische Disziplin Systemaspekte des vielfältigen phraseologischen Inventars einzelner Sprachen, so interessiert man sich aus funktional-pragmatischer Perspektive für Phraseme ${ }^{1}$ im Hinblick auf ihren (kon)textuellen Gebrauch, ihre Funktionen in typischen situativen und/oder textuellen Zusammenhängen, ihre potentiellen kommunikativen Leistungen, kurzum: man hinterfragt ihr semantisch-pragmatisches Potenzial. ${ }^{2}$ In der Tat lässt erst eine genaue Betrachtung kon- und kotextueller Umstände der Verwendung zuverlässige Aussagen über die in der Regel komplexe Semantik und Pragmatik von Phrasemen zu. Detailkenntnisse zum Bedeutungsspektrum und funktional-pragmatischen Potenzial einzelner phraseologischer Ausdrücke stellen zugleich eine erforderliche Basis für adäquate

vida.jesensek@um.si

1 In Anlehnung an Burger (2015: 30ff.) wird in diesem Beitrag das breite Verständnis der Phraseologie vertreten; unter Phrasem werden polylexikale, relativ stabile, satzglied- und/oder satzwertige Wortschatzeinhheiten mit graduell ausgeprägter Idiomatizität verstanden (den Ton angeben, blinder Passagier, klipp und klar, Übung macht den Meister). Sog. pragmatische Phraseme (Hyvärinnen 2011) bzw. Routineformeln (Stein 1995, Burger 2015) (guten Tag, mit freundlichen Grüßen, meines Erachtens) als phraseologische Sonderklasse werden dagegen nicht berücksichtigt.

2 Bekanntlich erweist sich eine klare Trennung zwischen Semantik und Pragmatik als äußerst schwierig, denn beide befassen sich mit Bedeutung. Gerade metaphorisch-metonymische (und somit phraseologische) Ausdrucksweisen stellen eine der Schnittstellen dar; es lässt sich nicht eindeutig festlegen, welche Bedeutungsaspekte eines (phraseologischen) Ausdrucks dem Bereich der Semantik und welche dem Bereich der Pragmatik zuzuordnen sind (Gutzmann/Schumacher 2018). Aus diesem Grund deckt der Ausdruck Pragmatik in diesem Beitrag immer auch Aspekte der Phrasem-Semantik. 
lexikographische Erfassung in allgemeinen und speziellen Wörterbüchern dar. Nicht minder wichtig sind sie aber auch in sprachdidaktischer und übersetzerischer Hinsicht: eine angemessene, sinnvolle und effiziente Vermittlung von Phraseologie im Sprachenlernen sowie eine adäquate Übersetzung sind ohne umfassende sprachkontrastive Kenntnisse über semantischen Gehalt und pragmatisches Potenzial eines jeden phraseologischen Ausdrucks nicht denkbar.

Der Beitrag ${ }^{3}$ wendet sich somit der Frage zu, wie man die komplexe Semantik und Pragmatik der Phraseologie lexikographisch erfasst, damit sowohl phraseologietheoretische als auch praktisch-lexikographische und wörterbuchbenutzerorientierte Ansprüche gedeckt werden. Somit wird das Ziel verfolgt, systematische, sprachkontrastive und empirisch gesicherte Detailuntersuchungen zur Semantik-Pragmatik der Phraseologie anzuregen. Die Thematik knüpft an die Forschungs- und Publikationstätigkeit des Jubilars, dem dieser Band gewidmet ist. Mein geehrter Doktorvater Herr Prof. Dr. Siegfried Heusinger widmete sich lange Zeit und intensiv den Fragen lexikaler Pragmatik. Durch seine akribisch-systematischen Einsichten in (kon)textuelle Zusammenhänge der sprachlichen Kommunikation hat er entscheidend auch zu meinen eigenen Forschungsinteressen beigetragen. Ich denke gern daran, wie er auf eine sanfte, jedoch entschlossene Art und Weise die Anfertigung meiner Doktorarbeit betreute und meine spätere akademische Laufbahn an der Universität Maribor stets mit regem Interesse verfolgte und unterstützend förderte. Dafür danke ich ihm von ganzem Herzen.

\section{PRAGMATISCHES IN DER PHRASEOLOGIE}

Bekannterweise sind Phraseme sowohl formal-strukturell als auch semantisch-pragmatisch komplexe Spracheinheiten. In der Regel ist ihre Semantik vielschichtig und mehrdimensional (Burger 2015: 61ff.), was u. a. mit dem sekundären semiotischen Charakter der Phraseologie einhergeht (ebenda: 78f.). Phraseme sind demnach Zeichen 2. Stufe; sie sind aus Zeichen zusammengesetzt, die selbst Zeichen 1. Stufe mit regulärer (extraphraseologischer) Bedeutung sind, während phraseologische Bedeutung die Bedeutung des ganzen Phrasems darstellt, die hauptsächlich im Grad der Idiomatizität ${ }^{4}$ einzelner Komponenten begründet ist.

Dazu enthalten Phraseme in der Regel kommunikativ-pragmatisches Potenzial. Es stellt sich die Frage, was man mit dem Phrasem tut, wenn man es in einem Text einsetzt und welche Wirkung damit beim Rezipienten evoziert wird/werden kann; aus diesem Blickwinkel seien sie „pragmatisch besonders geladen“ (Kühn 1994: 82). Demnach sind viele phraseologische Ausdrücke als Sprachmittel zur Realisierung spezifischer

3 Der Beitrag entstand im Rahmen der von der Slowenischen Forschungsagentur (ARRS) finanzierten Untersuchungen zur slowenischen Identität und Kultur in Sprachkontakträumen (P6-0372), Leiter der Forschungsgruppe M. Klemenčič.

4 Unter Idiomatizität versteht man in der Phraseologieforschung Verbindung der Bedeutung eines Phrasems mit der Bedeutung einzelner Komponenten, die allerdings sehr schwer (oder gar nicht) nachvollziehbar ist. In dieser Hinsicht wird oft der Begriff der semantischen Motivation/ Motiviertheit thematisiert. 
kommunikativer Aufgaben interpretierbar (Stein 1995), unter pragmatischen Aspekten lassen sich Phrasemen textuell-kommunikative Funktionen zuordnen. Es gilt, dass einzelnes Phrasem verschiedenartige Funktionen (gesondert oder gleichzeitig) erfüllen kann, die jedoch vom jeweiligen situativen Kontext abhängig sind und nur vor dessen Hintergrund erkannt und interpretiert werden können.

Über den pragmatischen Mehrwert von Phrasemen (Benennung nach Burger) diskutierte man bisher oft aus stilistischer Perspektive, meist im Hinblick auf die traditionell angenommene, jedoch vage und schwer bestimmbare Eigenschaft der Expressivität als Stilmerkmal (Burger 2015: 77ff.). Versteht man die Expressivität als eine latente Eigenschaft sprachlicher (auch phraseologischer) Ausdrücke, die unter bestimmten Bedingungen und in bestimmten Kontexten aktiviert wird, so spielen pragmatische Aspekte dabei eine Rolle, „die die konkrete Verwendung des Ausdrucks im Kontext bestimmen und seinen pragmatischen Mehrwert gegenüber quasi-synonymen nicht-phraseologischen Formulierungen ausmachen" (ebenda: 77). ${ }^{5}$

Auch wurden in der bisherigen Erforschung pragmatischer Aspekte der Phraseologie bewertende/qualifizierende Handlungen betont, die mit Phrasemen vollzogen werden (können); vgl. hierzu exemplarisch negative, abwertende bzw. positive, aufwertende Konnotationen der Ausdrücke ins Gras beißen, den Löffel abgeben bzw. sein Leben aushauchen, ins ewige Leben eingehen, aus dem Leben abberufen werden für ,sterben'. Bei Sprichwörtern spricht man von einer prinzipiellen polyfunktionalen und polysituativen Verwendung (u. a. Grzybek 1984, Lüger 1999, Mellado Blanco 2007, Burger 2015). Es werden hauptsächlich zwei funktional-pragmatische Handlungsaspekte festgelegt: Sprichwörter in sozialer Funktion gelten als Formulierungen von sozial anerkannten Überzeugungen, Werten und Normen (Grzybek 1984), Sprichwörter in kontextueller (pragmatischer) Funktion gelten in konkreten kommunikativen Situationen zugleich als Ausdruck von Sprachhandlungen wie Warnung, Überredung, Argument, Bestätigung, Trost, Besänftigung, Überzeugung, Mahnung, Zurechtweisung, Feststellung, Charakterisierung, Erklärung, Beschreibung, Rechtfertigung, Zusammenfassung (u. a. Röhrich/Mieder 1977, Kindt 2002, Jesenšek 2014a). Beide sind in einem wechselseitigen Verhältnis zu verstehen, denn »dass ein Sprichwort z. B. als Stütze in einer Argumentation verwendet werden kann (= kontextuelle Funktion), ist nur möglich, wenn es vom Sprecher und vom Hörer als Formulierung einer generellen Regel (= soziale Funktion) aufgefasst wird « (Burger 2015: 108). Zur Veranschaulichung: während Sprichwörter in (1) und (2) als expliziter Ausdruck soziokulturell konventionalisierter Werte und Normen gelten, ist das Sprichwort in (3) als Versprachlichung eines Ratschlags interpretierbar:

(1) Ich bin schon lange alleinerziehend und frage mich, ob ich vielleicht in manchen Dingen zu streng war. Erst die Arbeit und dann das Vergnügen, so bin ich erzogen worden. (SprichWort) ${ }^{6}$

5 Zu semantischen Relationen zwischen Phraseologie und Einwortlexik vgl. u. a. Proost (2007).

6 Typographische Hervorhebungen in zitierten Belegen stammen von der Autorin des Beitrags. 
(2) Lügen haben kurze Beine... so haben es die meisten schon in der Kindheit gelernt. Es lohnt sich nicht zu lügen, mit Lügen schadet man sich und andern. (SprichWort)

(3) „Sie sollten nicht mit zu viel Ehrgeiz an die Sache herangehen“, riet Jung den etwa 100 Zuhörern zu Beginn. Eile mit Weile sei vielmehr das Motto. Bevor der Anfänger oder Wiedereinsteiger überhaupt mit dem Sport beginne, sei erst einmal ein Gesundheitscheck fällig. (SprichWort)

Angesichts der Tatsache, dass Strukturen vieler Phraseme im Komponentenbestand variabel sind und folglich das definitorische Merkmal der strukturellen Stabilität nur eine relative Geltung hat, zeigt sich in vielen Fällen, dass sogar phraseologische Muster/Modelle, nach denen sich Strukturen vieler Phraseme richten, pragmatisch konventionalisiert sind. Vgl. hierzu das Muster eines sog. idiomatischen Satzes (Finkbeiner 2008, zit. nach Burger 2015: 58) Das ist (ja/doch) zum KONV (Konversion), wo textuelle Realisierungen die Pragmatik des Musters, nämlich AUSDRUCK EINER EMOTION, deutlich hervorbringen: Das ist (ja/doch) zum Verrücktwerden/In-dieLuft-Gehen/Aus-der-Haut-Fahren! Da Sprichwörter weitgehend Realisierungen syntaktischer Modellstrukturen und inhaltlicher Benennungsmodelle darstellen (Röhrich/ Mieder, 1977: 61f.), drängt die Modellhaftigkeit nahezu zur Bildung von Varianten und Modifikationen. Die Möglichkeit des variablen Umgangs mit Sprichwörtern wird bei den Sprechern oft wahrgenommen; insbesondere gilt das für textuelle Kontexte bzw. Textsorten, in denen das parömiologische Variabilitätspotenzial zu den jeweils aktuellen textuellen Funktionen und Sprecherintentionen gewinnbringend beiträgt, so z. B. in Werbetexten, Schlagzeilen und Titeln (Jesenšek 2014); vgl. die Modifikation des Sprichworts Ein Unglück kommt selten allein in der Betitelung in (4):

(4) »Ein Zwilling kommt selten allein«. Die romantische Familienkomödie mit Witz und Tempo! Eine Neuverfilmung von Erich Kästners Kinderbuchklassiker »Das doppelte Lottchen«. (SprichWort)

In solchen Zusammenhängen ist nicht zu übersehen, dass durch kreative Ausnutzung der möglichen Variabilität traditionell kollektivistische sprichwörtliche Aussagen zu individualistischen Ausdrucksweisen werden, im Einklang mit gegenwärtig aktuellen Individualisierungstendenzen ein Mittel zur Selbstdarstellung darstellen und somit das funktional-pragmatische Sprichwortpotenzial bereichern. Es leuchtet ein, dass Sprichwörter einen formalstrukturellen und funktional-pragmatischen Wandel erfahren. Fungierten sie einst als Instrument moralisch-erzieherischer Aufklärung, als rhetorisch-stilistisches Mittel und Indiz einer kultivierten Konversation, so zeigt sich ihre aktuelle Pragmatik vor allem in innovativ-kreativen, spielerischen textuellen Vorkommen, was in der Regel beabsichtigte stilistisch-pragmatische Auswirkungen mit sich bringt (u. a. Mieder 1999, Jesenšek 2014a, Burger 2015).

Relativ oft wird auch das textuelle Potenzial phraseologischer Ausdrücke erforscht (u. a. Lüger 1999, Umurova 2005, Richter-Vaapatalo 2007, Lewandowska 2008, 
Mellado Blanco 2008, Ptashnyk 2009, Hoffmann 2012, Valenčič Arh 2014, Pociask 2015). Untersuchungen zur Phraseologie in den Medien (Presse, Fernsehen, Internet), in der Werbung, in literarischen Texten (vor allem in der Kinder- und Jugendliteratur bzw. im literarischen Werk einzelner Autoren) betreffen in der Regel auch ihre textuelle Funktionalität. Textfunktionen, die durch den intendierten Einsatz der Phraseologie realisiert werden (können), werden bereits bei Koller (1977) festgelegt (Wertungs-, Anschaulichkeits-, Anbiederungs-, Vereinfachungs-, Argumentationsfunktion) und als typologisches Merkmal der Phraseologie interpretiert. Allerdings wurde schnell deutlich, dass nachvollziehbare Aussagen zum textuell-funktionalen Potenzial der Phraseologie erst durch eine systematische Berücksichtigung ko- und kontextueller Faktoren möglich ist; Verortung der Phraseme im Text, Phrasem-Modifikationen als intendierte formal-semantische Abwandlungen mit stilistisch-semantischen Effekten, Textsorte sind $u$. a. bedeutsame Untersuchungsaspekte, die in der bisherigen textuellen Erforschung der Phraseologie zu wichtigen Ergebnissen geführt haben.

Nach wie vor vermisst man allerdings ganzheitliche und betont pragmatische Forschungsansätze, die auf eine integrative Art und Weise die Ermittlung der in der Regel komplexen Semantik-Pragmatik phraseologischer Ausdrücke ermöglichen würden; der Feststellung Schafroths (Schafroth 2013: 188), dass es nie einen ganzheitlichen pragmalinguistischen Ansatz, Phraseologie zu untersuchen, gegeben hat, ist somit weitgehend zuzustimmen. Über Forschungsdesiderata in der Erfassung der phraseologischen Pragmatik berichtet ausführlich bereits Filatkina (2007: 152), wobei sie besonders „eine bessere empirische Basis und stärkere theoretische Aufbereitung“, textsortenvergleichende Studien zum Phraseologie-Gebrauch und praktische Umsetzung der Forschungsergebnisse vermisst. Dazu betont Schafroth (2013) bisher wenig bekannte kommunikative Effekte, die durch den Gebrauch von Phrasemen ausgelöst werden (können) und ihr Potenzial zur Selbstdarstellung des Sprechers. So ist auch die Beobachtung nicht besonders überraschend, dass die semantisch-pragmatische Darlegung von Phraseologie in allgemeinen und speziellen Wörterbüchern noch immer viel zu wünschen übrig lässt.

\section{PRAGMATIK DER PHRASEOLOGIE IN WÖRTERBÜCHERN}

Betrachtet man die Behandlung semantisch-pragmatischer Dimensionen phraseologischer Ausdrücke in gängigen ein- und mehrsprachigen allgemeinen und speziellen Wörterbüchern (mit Deutsch), so sind Ergebnisse nicht zufriedenstellend. Mehr noch: nach wie vor bleibt die lexikographische Erfassung der phraseologischen Semantik-Pragmatik das zentrale Problem der praktischen Lexikographie (u. a. Grzybek 1984, Kispál 2000 und 2007, Mieder 2003, Korhonen 2011, Jesenšek 2014 und 2018). Bereits kurze Einblicke in die gängige Praxis der sonst bedeutsamen Wörterbücher mit Deutsch (DWDS, Duden online, Duden 11) lassen erkennen, dass spezifische Aspekte der phraseologischen Semantik-Pragmatik wie etwa Idiomatizitätsgrad (Bildlichkeit), Expressivität, Kontextgebundenheit, Sprachhandlungspotenzial, Sprechereinstellungen, Textsorten- und Medienspezifik nicht systematisch betrachtet und erfasst werden. 
Beispiele zum Aspekt Idiomatizität:

(5) ins Gras beißen

DWDS: salopp, derb er musste ins Gras beißen (= sterben)

Duden online: ins Gras beißen (salopp: sterben; vermutlich nach der antiken Vorstellung, dass der Kämpfer beim Todeskampf in Erde oder Gras beißt)

Duden 11: ins Gras beißen (ugs.): (eines gewaltigen Todes) sterben

Das geplante Unternehmen war ihm viel zu gefährlich; er hatte keine Lust, ins Gras zu beißen.

Der Soldat H. hatte hingegen einer Einheit angehört, bei der es nicht üblich war, ins Gras zu beißen.

(6) den Löffel angeben

DWDS: salopp den Löffel abgeben (= sterben)

Duden online: den Löffel sinken lassen/fallen lassen/hinlegen/wegwerfen/ wegschmeißen/abgeben (salopp: sterben)

Duden 11: den Löffel abgeben/hinlegen/fallen lassen/wegschmeißen (salopp verhüll.: sterben)

Der Soldat stirbt auch nicht, er fällt, oder er hat den Löffel weggeschmießen. ...ich hätte nie im Leben freiwillig den Löffel abgegeben.

Der Löffel steht in dieser Wendung für die lebensnotwendige Tätigkeit des Essens

Man beobachtet vereinfachte (reduzierte) Erfassung der Bedeutung (Wiedergabe mit dem Einwortlexem), teilweise uneinheitliche Stilschichtmarkierung, kulturhistorische Remotivation ist nur im speziellen phraseologischen Wörterbuch vorhanden, Beispiele fehlen oder sie sind für Zwecke der Bedeutungserschließung weniger hilfreich, Synonymierelationen werden nicht erfasst.

Zum Bedeutungsaspekt Expressivität:

(7) etw. an den Mann bringen

DWDS: umgangssprachlich 〈etw. an den Mann bringen〉 etw. loswerden Beispiele: eine Ware an den Mann bringen; sie suchte eine Gelegenheit, ihre Weisheit, die Neuigkeit an den Mann zu bringen

Duden online: etwas an den Mann bringen (umgangssprachlich: 1. etwas verkaufen. 2. im Gespräch o. Ä. etwas mitteilen, äußern, erzählen)

Duden 11: etwas an den Mann bringen (ugs.): etw. verkaufen: Noch schwerer als die Wohnungen sind offenbar die gewerblichen Räume an den Mann zu bringen

Bedeutungsdifferenzierung und -paraphrasen sind uneinheitlich (loswerden, verkaufen), erst durch Belege/Beispiele ist die Ausdrucksstärke (im Sinne der Expressivität, i. e. einer positiven Bewertung des Agens durch den Sprecher) gegenüber verkaufen/mitteilen nachvollziehbar. 
Zum Bedeutungsaspekt Kontextgebundenheit:

(8) neue Besen kehren gut

DWDS: sprichwörtlich neue Besen kehren gut (= wer eine Stelle antritt, zeigt sich anfangs besonders eifrig)

Duden online: neue Besen kehren gut (wenn jemand etwas Neues in Angriff nimmt, macht er es anfangs mit besonderem Eifer)

Duden 11: neue Besen kehren gut: wenn jmd. eine neue Aufgabe o. Ä. übernimmt, zeigt er anfänglich besonderen Eifer: Neue Besen aber kehren gut, und Konvertiten übertreffen die Altgläubigen zumeist an Eifer

Bedeutungsparaphrasen weisen auf den inhaltlichen Kontext hin (Kontext der Arbeit, Anfang von etw. Neuem), allerdings unvollständig (neue Ideen, (positive) Veränderungen, Wechsel in der Führungsposition, auch Zweifel an Neuem).

Zum Bedeutungsaspekt Sprachhandlungspotenzial:

(9) junge/jüngere Beine haben

DWDS: du kannst stehen, du hast noch junge Beine (= bist noch jung)

Duden online: jüngere Beine haben (umgangssprachlich: besser als eine ältere Person laufen können)

Duden 11: jüngere Beine haben (ugs.): besser als ein Älterer laufen können Kannst du das nicht erledigen? Du hast doch jüngere Beine

(10) vor seiner eigenen Tür kehren

DWDS: jeder kehre vor seiner Tür (= jeder kümmere sich um seine eigenen Schwächen)

Duden online: vor seiner eigenen Tür kehren (umgangssprachlich: sich um seine eigenen Angelegenheiten kümmern)

Duden 11: vor seiner eigenen Tür kehren/fegen: die eigenen Fehler ablegen, bevor man andere kritisiert

Europa muss auch vor der eigenen Tür kehren. Es muss seine Verantwortung für die Geschichte und Gegenwart der europäisch-afrikanischen Beziehungen annehmen

Man sieht bei (9) eine Verallgemeinerung/Reduktion der Bedeutung (,jung sein ${ }^{6}$ ), Remotivation (laufen können), die illokutive Komponente AUFFORDERUNG bzw. VORSCHLAG ZUR HILFE wird nur durch Beispiel impliziert. Bei (10) überraschen uneinheitliche Bedeutungsparaphrasen (Schwäche, Angelegenheit, Fehler), die illokutive Komponente AUFFORDERUNG bzw. RAT wird durch die grammatischen Formen (Konj., Modalverb) angedeutet.

$\mathrm{Zu}$ Aspekten Sprechereinstellungen und Handlungspotential: 


\section{(11) sich etw. aus dem Kopf schlagen}

Duden online: sich $<$ Dativ $>$ etwas aus dem Kopf schlagen (einen Plan o. Ä. aufgeben)

Duden 11: sich etw. aus dem Kopf schlagen (ugs.): ein Vorhaben aufgeben Sie müssen sich das aus dem Kopf schlagen. Sie ist kein Mädchen, das mit einem Freund gehen könnte

Die stark vereinfachte Erfassung der Bedeutung betrifft vor allem fehlende explizite Angaben zu negativen Sprechereinstellungen (ablehnend, etw. wird als unrealisierbar, unpassend, unangebracht, unrealistisch verstanden); das Handlungspotential (AUFFORDERUNG) ist teils durch Beleg/Beispiel erkennbar (müssen).

Kurzum: die lexikographische Behandlung der komplexen phraseologischen Semantik-Pragmatik ist uneinheitlich, unvollständig, vereinfachend; Bedeutungsparaphrasen sind, falls vorhanden, auf Denotatives bezogen, nichtdenotative, also pragmatische Bedeutungskomponenten werden in der Regel nicht expliziert, eventuell sind sie an den Belegen/Beispielen nachvollziehbar.

\section{SCHLUSSFOLGERUNG}

Aus den obigen Ausführungen ergeben sich mehrere Schlussfolgerungen:

- Pragmatischer Mehrwert von Phraseologie kann in einem Wörterbuch nur durch das sog. gebrauchspragmatische Konzept der lexikographischen Bedeutungserfassung sichtbar werden (Kühn 2004: 147). Damit ist ein holistischer, ganzheitlicher Zugang zur Bedeutung gemeint, indem »die Bedeutung eines Sprachzeichens als Formulierungen seiner Verwendungsregeln verstanden wird.« Bedeutung ist demnach Formulierung von Gebrauchsregeln, Gebrauchsregeln sind Referenz- und/oder Prädikationsregeln, pragmatisch-situative Verwendungsregeln oder eine Kombination davon. Im Fall der Phraseologie wird somit die Gebrauchsspezifik samt gesamtem pragmatischen, textuellen und assoziativen Potenzial von Phrasemen berücksichtigt.

- Der Angabenbereich zur Semantik-Pragmatik der Phraseologie ist in einem konkreten Wörterbuch typologisch und funktional determiniert und durch Vielfalt gekennzeichnet. Angaben betreffen denotative und nicht-denotative Bedeutungsaspekte, darunter illokutives und textbildendes Potenzial, präferierte und/ oder restringierte Verwendung in bestimmten Situationen und Kontexten, kommunikativen Domänen, Textsorten, eventuelle themengebundene Verwendung u. a. m.

- Das gebrauchspragmatische Konzept der lexikographischen Bedeutungserfassung hat methodisch-methodologische Folgen, denn eine derartige mehrdimensionale Erschließung und Erfassung der Bedeutung ist nur anhand der Beobachtung von tatsächlicher Sprachverwendung möglich. Systematische Auswertung von Sprachkorpora ist unerlässlich.

- Durch die korpusbasierte und mehrdimensionale lexikographische Erfassung der phraseologischen Bedeutung soll für den Wörterbuchbenutzer Folgendes 
erschließbar sein: Wissen über das denotative Bedeutungspotenzial einzelner Phraseme, Wissen über das textuelle Verhalten (inkl. phrasemspezifische Aspekte der Pragmatik), Wissen über die semantischen Relationen einzelner Phraseme zu anderen (phraseologischen und nichtphraseologischen) Wortschatzeinheiten.

- Schließlich soll die komplexe Semantik-Pragmatik der Phraseologie lexikographisch so dargestellt werden, dass die Kluft zwischen dem Geschriebenem und dem Verstandenem/ Interpretiertem von Seiten des Benutzers kleinstmöglich ist (Bielińska 2018).

Eine gebrauchspragmatisch konzipierte lexikographische Erfassung der phraseologischen Semantik-Pragmatik ist in (12) dargestellt. Durch die Angabenstruktur wird verstehens- und verwendungsrelevantes phraseologisches Wissen vermittelt, es werden neue Anforderungen an die Sprachdidaktik gestellt (Förderung der aktiven phraseologischen Kompetenz) und es sollen Übersetzer bei der Suche nach adäquaten Übersetzungen unterstützt werden. Das Beschreibungsmodell wurde in einem EU-Forschungsprojekt entwickelt und in der Form einer mehrsprachigen phraseologischen Datenbank umgesetzt. ${ }^{7}$ Methoden und Angabenstrukturen sind übertragbar und anpassungsfähig und es ist zu wünschen, dass sie zu vertieften sprachkontrastiven Untersuchungen der Phraseologie und folglich zu besseren lexikographischen Praktiken anregen werden.

\section{(12) In der Ruhe liegt die Kraft}

Sagt man dafür, dass man mit Gelassenheit und bedachtem Vorgehen oft mehr erreichen kann als mit überstürtztem Handeln oder hektischer Betriebsamkeit.

Das Sprichwort ist in der Grundaussage, dass ausreichend Zeit wichtig für den Erfolg einer Sache ist, ein Synonym zu Eile mit Weile, Gut Ding braucht Weile, Rom wurde auch nicht an einem Tag erbaut, Geduld bringt Rosen.

In den Korpusbelegen wird mit dem Sprichwort häufig

- der Rat erteilt, bei Problemen gelassen bzw. geduldig zu bleiben, u. a. in Horoskopen,

- die Wichtigkeit einer gelassenen Lebenshaltung und der Schaffung ruhiger Momente in der modernen Gesellschaft, die von Druck und Hektik geprägt ist, betont,

- $\quad$ auf meditative Situationen, die der Entspannung dienen, Bezug genommen,

- $\quad$ ein Lebensmotto von Menschen bezeichnet,

- auf Gelassenheit als Grund für Erfolge im Sport verwiesen. (SprichWort)

7 SprichWort-Plattform. 2008-2010. Projektleiterin Vida Jesenšek. http://www.sprichwortplattform.org/. 


\section{Literatur}

BIELIŃSKA, Monika (2018) „Einige Überlegungen zu lexikographischen Kohärenzstrukturen.“ In: V. Jesenšek/M. Enčeva (Hrsg.), Wörterbuchstrukturen zwischen Theorie und Praxis. Berlin: de Gruyter, 73-84.

BURGER, Harald (2007) „Semantic Aspects of Phrasemes.“ In: H. Burger et al. (Hrsg.), 90-109.

BURGER, Harald (2015) Phraseologie: Eine Einführung am Beispiel des Deutschen. 5. Aufl. Berlin: Erich Schmidt.

BURGER, Harald et al. (Hrsg.) (2007) Phraseologie: Ein internationales Handbuch der zeitgenössischen Forschung. Berlin/New York: de Gruyter.

FILATKINA, Natalia (2007) „Pragmatische Beschreibungsansätze.“ In: H. Burger et al. (Hrsg.), 132-158.

FINKBEINER, Rita (2008) Idiomatische Sätze im Deutschen: Syntaktische, semantische und pragmatische Studien und Untersuchung ihrer Produktivität. Stockholm: Stockholm University Press.

GRZYBEK, Peter (1984), Zur lexikographischen Erfassung von Sprichwörtern.“ In: P. Grzybek/W. Eismann (Hrsg.), Semiotische Studien zum Sprichwort-Simple Forms Reconsidered I. Kodikas Code Ars Semeiotica. An International Journal of Semiotics 3/4, 345-350.

GUTZMANN, Daniel/Petra B. SCHUHMACHER (2018) „Schnittstelle Semantik-Pragmatik.“ In: A. Wöllstein et al. (Hrsg.), Grammatiktheorie und Empirie in der germanistischen Linguistik. Berlin/Boston: de Gruyter, 471-509.

HEUSINGER, Siegfried (1995) Pragmalinguistik. Texterzeugung, Textanalyse; Stilgestaltung und Stilwirkungen in der sprachlichen Kommunikation. Ein Lehr- und Übungsbuch. Frankfurt a. Main: Haag und Herchen.

HOFFMANN, Sarah (2012) Argumentative Strukturen in Sprichwörtern. Bern [etc.]: Peter Lang.

HYVÄRINNEN, Irma (2011), ZZur Abgrenzung und Typologie pragmatischer Phraseologismen - Forschungsüberblick und offene Fragen. “ In: I. Hyvärinnen/A. Liimatainen (Hrsg.), Beiträge zur pragmatischen Phraseologie. Frankfurt a. Main: Peter Lang, 9-43.

JESENŠEK, Vida (2014) „Frazeologija v spletni leksikografiji.“ In: V. Jesenšek (Hrsg.), Frazeologija nemškega jezika z vidikov kontrastivnega in uporabnega jezikoslovja/Phraseology of the German language from the perspective of contrastive and applied linguistics. Maribor: Filozofska fakulteta, Oddelek za germanistiko, 338-361.

JESENŠEK, Vida (2014a) „Pragmatic and stylistic aspects of proverbs.“ In: H. Hrisztova-Gotthardt/M. A. Varga (Hrsg.), Introduction to paremiology. A comprehensive guide to proverb studies. Warsaw/Berlin: de Gruyter, 133-161.

JESENŠEK, Vida (2018) „Korpusempirische Sprachdaten in der Parömiographie: Potenzial und Grenzen.“ In: A. Gondek/A. Jurasz/J. Szczek (Hrsg.), Einblicke, Rückblicke: Beiträge zur deutschen Phraseologie und Parömiologie aus intra- und interlingualer Sicht. Bd. 1. Baltmannsweiler: Schneider Verlag Hohengehren, 59-74. 
KINDT, Walther (2002) „Kommunikative Funktionen von Sprichwörtern: Ein Beispiel für die notwendige Verbindung von Phraseologie und Pragmatik." In: E. Piirainen/I. T. Piirainen (Hrsg.), Phraseologie in Raum und Zeit: Akten der 10. Tagung des Westfälischen Arbeitskreises Phraseologie/Parömiologie (Münster, 2001). Baltmannsweiler: Schneider Verlag Hohengehren, 273-286.

KISPÁL, Tamás (2000) „Sprichwörter in einem phraseologischen Wörterbuch.“ InfoDaF 27, 367-375.

KISPÁL, Tamás (2007) „Sprichwörtersammlungen.“ In: H. Burger et al. (Hrsg.), 414-423.

KOLLER, Werner (1977) Redensarten: Linguistische Aspekte, Vorkommensanalysen, Sprachspiel. Tübingen: Niemeyer.

KORHONEN, Jarmo (2011) Phraseologie und Lexikografie: Phraseologismen in einund zweisprachigen Wörterbüchern mit Deutsch. Burlington, Vermont: The University of Vermont.

KÜHN, Peter (1994) „Pragmatische Phraseologie. Konsequenzen für die Phraseographie und Phraseodidaktik.“ In: B. Sandig (Hrsg.), Europhras 92: Tendenzen der Phraseologieforschung. Bochum: Brockmeyer, 411-428.

LEWANDOWSKA, Anna (2008) Sprichwort-Gebrauch heute. Ein interkulturell-kontrastiver Vergleich von Sprichwörtern anhand polnischer und deutscher Printmedien. Bern: Peter Lang.

LÜGER, Heinz-Helmut (1999) Satzwertige Phraseologismen: Eine pragmalinguistische Untersuchung. Wien: Praesens.

MELLADO BLANCO, Carmen (2007) „Die nicht denotativen Bedeutungskomponenten der Phraseologismen: ihre phraseographische Behandlung im Rahmen der strukturellen Semantik." Deutsche Sprache 35, 315-333.

MELLADO BLANCO, Carmen (Hrsg.) (2008) Beiträge zur Phraseologie aus textueller Sicht. Hamburg: Verlag Dr. Kovac.

MIEDER, Wolfgang (1999) „Sprichwörter des Kontinents.“In: W. Köpke/B. Schmelz (Hrsg.), Das gemeinsame Haus Europa. Handbuch zur europäischen Kulturgeschichte. München: Deutscher Taschenbuch-Verlag, 956-965.

MIEDER, Wolfgang (2003) „Sprichwörter im GWDS.“ In: H. E. Wiegand (Hrsg.), Untersuchungen zur kommerziellen Lexikographie der deutschen Gegenwartssprache I. Duden. Das große Wörterbuch der deutschen Sprache in zehn Bänden. Print-und CD-ROM-Version. Tübingen: Niemeyer, 413-436.

POCIASK, Janusz (2015) „Phraseologismen in deutschen und polnischen Pressetexten, dargestellt aus pragmalinguistischer Perspektive." Linguistik online 74, 5/15, $97-117$.

PROOST, Kristel (2007) „Paradigmatic relations of phrasemes.“ In: H. Burger et al. (Hrsg.), 110-119.

PTASHNYK, Stefaniya (2009) Phraseologische Modifikationen und ihre Funktionen im Text. Eine Studie am Beispiel der deutschsprachigen Presse. Balltmannsweiler: Schneider Hohengehren. 
RICHTER-VAPAATALO, Ulrike (2007) Da hatte das Pferd die Nüstern voll. Gebrauch und Funktion von Phraseologie im Kinderbuch. Frankfurt a. Main: Peter Lang.

RÖHRICH, Lutz, Wolfgang MIEDER (1977) Sprichwort. Stuttgart: Metzler.

SANDIG, Barbara (2007) „Stilistische Funktionen von Phrasemen.“ In: H. Burger et al. (Hrsg.), 158-175.

SCHAFROTH, Elmar (2013) „Das pragmatische Potential von Phrasemen - illustriert am Deutschen und Italienischen.“'In: S. Cantarini (Hrsg.), Wortschatz, Wortschätze im Vergleich und Wörterbücher: Methoden, Instrumente und neue Perspektiven. Frankfurt a. Main: Peter Lang, 185-208.

SPRICHWORT. SprichWort-Plattform. www.sprichwort-plattform.org [13.11.2018]. STEIN, Stefan (1995) Formelhafte Sprache. Untersuchungen zu ihren pragmatischen und kognitiven Funktionen im gegenwärtigen Deutsch. Frankfurt a. Main [etc.]: Peter Lang.

UMUROVA, Gulnas (2005) Was der Volksmund in einem Sprichwort verpackt ... : moderne Aspekte des Sprichwortgebrauchs anhand von Beispielen aus dem Internet. Frankfurt a. Main etc.: Peter Lang.

VALENČIČ ARH, Urška (2014) „Ein Prinz auf der Erbse“. Phraseologie und Übersetzung: Am Beispiel der Kinder- und Jugendliteratur von Christine Nöstlinger im Deutschen und Slowenischen. Balltmannsweiler: Schneider Hohengehren.

\section{Zusammenfassung PHRASEOLOGIE UND PRAGMATIK}

Der Beitrag geht von der Beobachtung aus, dass die semantisch-pragmatische Darlegung von Phraseologie in allgemeinen und speziellen Wörterbüchern noch immer viel zu wünschen übrig lässt. Er wendet sich der Frage zu, wie man die komplexe phraseologische Semantik und Pragmatik lexikographisch erfasst, damit sowohl phraseologietheoretische als auch praktisch-lexikographische und wörterbuchbenutzerorientierte Ansprüche gedeckt werden. Speziell geht er auf ausgewählte Aspekte der Pragmatik phraseologischer Ausdrücke ein (Bildlichkeit, Expressivität, Kontextgebundenheit, Sprachhandlungspotenzial, Sprechereinstellungen, Textsorten- und Medienspezifik), um exemplarisch zu zeigen, welche Anforderungen sich daraus für die (kontrastive) Phraseologie, praktische Lexikographie, Sprachdidaktik und übersetzerische Praxis ergeben. Vor allem wird dadurch das Ziel verfolgt, systematische, korpusempirische, analytische und sprachkontrastive Detailuntersuchungen zur Pragmatik der Phraseologie anzuregen und intensivieren. Zugleich werden Probleme angesprochen, die das in der Regel reiche pragmatische Potenzial der Phraseologie sowohl für die theoretische Phraseologieforschung als auch für praktische Anwendung deren Ergebnisse darstellt.

Schlüsselwörter: Phraseologie, Pragmatik, Lexikographie, Sprachdidaktik, Übersetzung 


\section{Abstract \\ PHRASEOLOGY AND PRAGMATICS}

The article has as its starting point the observation that the semantic-pragmatic presentation of phraseology in general and specialized dictionaries still tends to be inadequate. It tackles the question of how the complex semantics and pragmatics of phraseological units should be lexicographically represented, so that both the theoretical and practical lexicographical aspects as well as the user-oriented needs are appropriately considered. In particular, it addresses selected aspects of the pragmatics of phraseological units (figurativity, expressivity, contextuality, speech act potential, speaker attitude, text-type and media specificity) to exemplify which are the requirements for (contrastive) phraseology, applied lexicography, language didactics and translational practice. The article's primary goal is to encourage and facilitate detailed systematic, corpus-empirical, analytical and contrastive explorations of the pragmatic aspects of phraseology. At the same time, it touches upon the problems that the rich pragmatic potential of phraseology poses for both theoretical phraseology and the practical application of its findings.

Key words: phraseology, pragmatics, lexicography, language didactics, translation

\section{Povzetek \\ FRAZEOLOGIJA IN PRAGMATIKA}

Prispevek izhaja iz ugotovitve, da je leksikografska obravnava kompleksnih semantičnih in pragmatičnih lastnosti tipološko raznolikega frazeološkega izrazja $\mathrm{v}$ splošnih in specialnih slovarjih (še vedno) pomanjkljiva. Ukvarja se z vprašanjem, kako v slovarjih celovito zajeti pomenske in pragmatične razsežnosti frazeologije in hkrati zadostiti pričakovanjem, potrebam in zahtevam teoretične frazeološke in leksikografske vede, praktične leksikografije in predvidenih slovarskih uporabnikov. Obravnavani so nekateri pragmatični vidiki, ki pogosto določajo in vidno zaznamujejo besedilno rabo frazeološkega izrazja (slikovitost, ekspresivnost, vpetost v širši sporočanjski kontekst, zmožnost izražanja govornih dejanj in govorčevih stališč, besedilnovrstne in prenosniške posebnosti), eksemplarično je opozorjeno na potrebo po njihovi podrobni obravnavi v okvirih teoretske (kontrastivne) frazeologije, praktične leksikografije, jezikovne didaktike in prevajalske prakse, poudarjena je potreba po metodološko jasnih, natančnih, korpusno empiričnih, sistematičnih, analitičnih in kontrastivnih raziskavah pragmatike frazeološkega izrazja. Ob tem so v prispevku izpostavljene nekatere težave, ki jih le-ta povzroča pri teoretičnem raziskovanju frazeologije in praktično leksikografski uporabi njenih izsledkov.

Ključne besede: frazeologija, pragmatika, leksikografija, jezikovna didaktika, prevajanje 